\title{
Dopant-Induced Crossover from 1D to 3D Charge Transport in Conjugated Polymers
}

\author{
J. A. Reedijk, H. C.F. Martens, and H. B. Brom \\ Kamerlingh Onnes Laboratory, Leiden University, P.O. Box 9504, 2300 RA Leiden, The Netherlands \\ M. A. J. Michels \\ Department of Applied Physics, Eindhoven University of Technology, and Dutch Polymer Institute, \\ P.O. Box 513, 5600 MB Eindhoven, The Netherlands
}

(Received 29 April 1999)

\begin{abstract}
The interplay between inter- and intrachain charge transport in bulk polythiophene in the hopping regime has been clarified by studying the conductivity $\sigma$ as a function of frequency $\omega / 2 \pi$ (up to $3 \mathrm{THz}$ ), temperature $T$, and doping level $c$. We present a model which quantitatively explains the observed crossover from quasi-one-dimensional transport to three-dimensional hopping conduction with increasing doping level. At high frequencies the conductivity is dominated by charge transport on onedimensional conducting chains.
\end{abstract}

PACS numbers: 71.20.Rv, 71.55.Jv, 72.60. $+\mathrm{g}$, 72.80.Le

The charge transport mechanisms in conjugated polymers, although extensively studied over the past two decades, are still far from being completely understood. Neither the behavior around the insulator-to-metal transition (IMT), which can be induced in several polymer materials upon appropriate doping, nor the nature of hopping transport in the deeply insulating regime is yet resolved. While some studies indicate that transport is dominated by hops between three-dimensional (3D), well conducting regions [1,2], in other cases the strongly one-dimensional (1D) character of the polymer systems appears to be a crucial factor [3-5].

In investigating the nature of hopping transport in conjugated polymers, studying the temperature and doping level dependence of the dc conductivity is an important tool. Since the dc conductivity is determined by the weakest links in the conducting path spanning the sample, the study of $\sigma_{\mathrm{dc}}(T)$ gives insight in the slowest relevant transport processes in the system.

On the insulating side of the IMT, the dc conductivity is predicted by many models to follow the well-known hopping expression

$$
\sigma_{\mathrm{dc}}=\sigma_{0} e^{-\left(T_{0} / T\right)^{\gamma}}
$$

where the value of $\gamma$ and the interpretation of $T_{0}$ depend on the details of the model. The original Mott theory for 3D variable range hopping with a constant density of states (DOS) at the Fermi energy predicts $\gamma=1 / 4$ [6], while several modifications of the model have been proposed to describe the frequently observed value $\gamma=1 / 2$. Studying the dependence of $\gamma$ and $T_{0}$ on doping level $c$ provides the opportunity to discriminate between the various hopping models and extract parameters determining the conductive properties such as the DOS and the localization length.

While the dc conductivity is sensitive to the slowest transport processes, the ac conductivity $\sigma(\omega)$ provides information about processes occurring at time scales $\tau \approx \omega^{-1}$. Especially in conjugated polymers, where intrachain and interchain transition rates may differ by orders of magnitude, knowledge of $\sigma(\omega)$ at high frequencies can help to clarify the properties of charge transport on a polymer chain.

In this Letter, we present a systematic study of the charge transport in a conjugated polymer far away from the IMT, as a function of frequency, temperature, and doping level. By selecting a polymer system with very low interchain mobility, a separation of interchain and intrachain contributions to the conductivity can be made when the applied frequency is varied over 12 decades. At low frequencies, transport between chains is studied, while at frequencies well above the interchain transition rate, intrachain conduction is probed.

Experimental. - The experiments were performed on disks (thickness between 0.4 and $1.0 \mathrm{~mm}$ ) of pressed powders of the conjugated polymer poly $(3,4-\mathrm{di}-[(R, S)$ 2-methyl-butoxy]thiophene), abbreviated as PMBTh, the synthesis of which is described elsewhere [7]. The samples were doped with $\mathrm{FeCl}_{3}$ in a dichloromethane solution at doping levels $0.01<c<0.22$; here $c$ is the number of doped carriers per thiophene ring, which was determined with the aid of Mössbauer spectroscopy [8,9]. After doping, the solvent was evaporated and the resulting powders were vacuum dried overnight. The conductivity of the samples remained unchanged in an ambient atmosphere for several weeks. Contact resistances were less than $5 \%$ of the sample resistance [10]. The conductivity data were taken in the range $5 \mathrm{~Hz}$ through $3 \mathrm{THz}$ with the aid of several experimental methods, which are described elsewhere [11]. The conductivity in the range $0.3-3 \mathrm{THz}$ was determined from the transmission measured with a Bruker Fourier transform infrared spectrometer.

$d c$ conductivity. - The temperature-dependent static conductivity of samples with doping levels ranging from 0.01 to 0.22 is plotted in Fig. 1 . Here, $\log \sigma_{\mathrm{dc}}$ is plotted vs $T^{-1 / 2}$, so that data sets following Eq. (1) with 


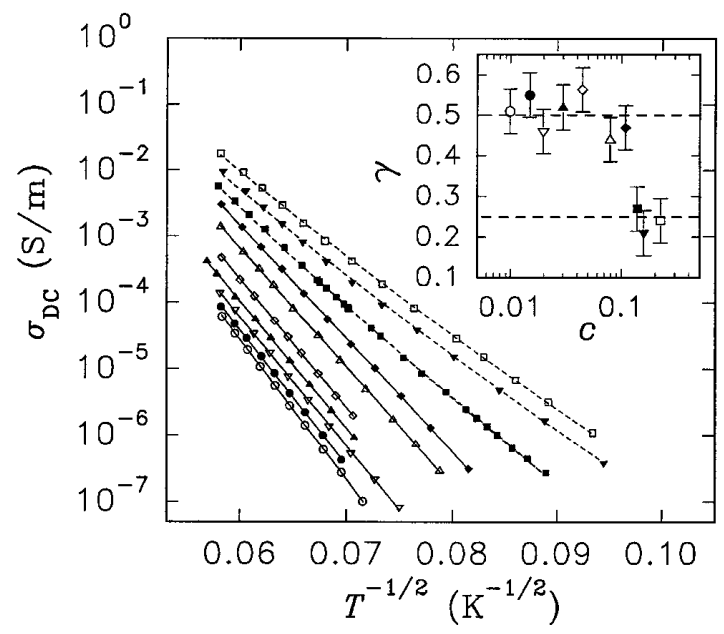

FIG. 1. dc conductivity as a function of temperature for several doping levels. The data are fitted to $\sigma_{\mathrm{dc}}=$ $\sigma_{0} \exp \left[-\left(T_{0} / T\right)^{\gamma}\right]$. For the solid lines $\gamma=1 / 2$, whereas for the dashed lines $\gamma=1 / 4$. The inset shows the $c$ dependence of $\gamma$ and defines the symbols used in this graph.

$\gamma=1 / 2$ fall on a straight line. In the inset, the value of $\gamma$ is plotted vs $c$. The exponent $\gamma$ has been determined by plotting the logarithm of the reduced activation energy $W=d \ln \sigma / d \ln T$ versus $\ln T$ and fitting it to a straight line; the slope of this line gives $\gamma$ [12]. The data show a clear transition in the dc behavior as a function of doping level around $c_{0}=0.12$. For low doping $c<c_{0}$, $\gamma$ values are grouped around $1 / 2$, whereas for $c>c_{0}$, $\gamma$ is close to the Mott value $1 / 4$. The conductivity data for $c<c_{0}$ are now fitted to Eq. (1) with $\gamma$ fixed at $1 / 2$ (solid lines), while the data for $c>c_{0}$ are fitted with a fixed $\gamma=1 / 4$ (dashed lines). Note that as the conductivity is many of orders of magnitude below the minimum metallic conductivity $\sim 100 \mathrm{~S} / \mathrm{cm}$ and depends very strongly on $T$, PMBTh is far away from the IMT at all doping levels.

Many authors [2-5] have reported the conductivity in conjugated polymers to follow Eq. (1) with $\gamma=1 / 2$, and several models have been proposed to explain this value. In disordered systems, the single particle DOS around the Fermi energy has a parabolic shape when longrange Coulomb interactions between charge carriers are dominant [13]. Inserting a quadratic DOS in the original Mott argument yields the exponent $\gamma=1 / 2$. Data on various conjugated polymers close to the IMT have been interpreted within this Efros and Shklovskii Coulomb-gap model [14]. Alternatively, it has been argued that polymer materials can be viewed as 3D granular metallic systems when the strong, inhomogeneous disorder in polymer materials leads to the formation of well conducting 3D regions separated by poorly conducting barriers [1,2]. For granular metals, $\sigma_{\mathrm{dc}}(T)$, though still not completely understood, is widely accepted to follow Eq. (1) with $\gamma=1 / 2$ [15]. Close to the IMT, both models predict a crossover from $\gamma=1 / 2$ to $\gamma=1 / 4$. Such transitions have indeed been observed in a number of systems $[14,16,17]$. However, because PMBTh is far away from the IMT at all $c$, these models cannot be applied here.

To explain our data we will use another approach, which is an extension of the quasi-1D hopping model of Nakhmedov et al. [18]. In their model, the charge carriers are supposed to be strongly localized on single chains (or 1D bundles of chains). Variable-range hopping is possible along the chains, while perpendicular to the chains with vanishingly small interchain overlap only nearest-neighbor hopping is allowed. Although the quasi1D model predicts $\gamma=1 / 2$ strictly speaking only for the anisotropic effective conductivity perpendicular to the chains, it has been successfully applied to randomly oriented polymer systems [3]. In a closely related approach, the polymer system is viewed as a fractal structure with a fractal dimension $d_{f}$ slightly greater than one [19]. The static conductivity on such a nearly $1 \mathrm{D}$ fractal is also calculated to follow Eq. (1) with $\gamma=1 / 2$.

Model. - To include a transition from quasi-1D hopping to 3D hopping as a function of doping level, the models mentioned above need to be extended. In the quasi-1D model such a transition is expected when the transverse overlap is sufficiently increased. In the nearly $1 \mathrm{D}$ fractal model, an increase in the fractal dimension would eventually lead to a decrease of $\gamma$ down to $1 / 4$. So in both cases, a transition to 3D hopping is induced by an increase of the interchain connectivity. Before we present our calculations, let us show how this can be qualitatively understood. Upon chemically doping a conjugated polymer, not only charge carriers but also dopant counterions are added to the system. The counterions locally decrease the interchain potential seen by the carriers, and thus considerably enhance the hopping rate [2]. Since the Fermi energy is shifted upwards upon doping, the counterions must even be considered as hopping sites when the distance of the dopant site energy to the Fermi level becomes of the order of the hopping energy. In this view, the sharply defined crossover at $c_{0}$ can be interpreted as the point where the dopant sites start to play an active role in the hopping process, thereby enhancing the density of hopping sites and making variable range hopping in the direction perpendicular to the chains possible.

We now show quantitatively that the conductivity in both doping regimes and the crossover with doping level can be explained in a single variable-range hopping model. We assume hops within an energy interval $E$ over a distance $(X, Z)$ in the combined parallel $(x)$ and orthogonal $(z)$ directions, with localization lengths $L_{x}$ and $L_{z}$, respectively. For an electron on a chain, the local DOS $n$ contains two contributions, $n_{0}$ from the chain itself and $n_{1}$ from the neighboring chains and from intermediate dopant sites. As the latter contribution depends stronger on $c$ than the former, $n_{1} / n_{0}$ rises with doping level. Following the usual variable-range hopping arguments, the conductivity 
$\sigma \propto \exp \left[-2 X / L_{x}-2 Z / L_{z}-E / k_{B} T\right]$ should be maximized under the condition $2 X(2 Z)^{2}\langle n\rangle E \approx 1$, where $\langle n\rangle$ is the DOS averaged over the volume $(2 X, 2 Z, 2 Z)$, for which we write $\langle n\rangle=n_{0}\left(L_{z} / 2 Z\right)^{2}+n_{1}$ [20]. We introduce $\xi=2 X / L_{x}, \zeta=2 Z / L_{z}$, and $\epsilon=E / k_{B} T$ and note that $\langle n\rangle$ does not depend on $X$, leading to $\epsilon=\xi$, i.e., $\sigma \propto \exp [-2 \xi-\zeta]$. Optimizing in the "high doping" limit $\zeta^{2} \gg n_{0} / n_{1}$, we find $2 \xi=\zeta$ and $\xi^{-4}=4 k_{B} T L_{x} L_{z}^{2} n_{1}$, which gives $\sigma \propto \exp [-4 \xi]=$ $\exp \left[-\left(T_{0}^{\text {high }} / T\right)^{1 / 4}\right]$ with $T_{0}^{\text {high }}=64 /\left(k_{B} L_{x} L_{z}^{2} n_{1}\right)$. For the "low-doping" regime $\zeta^{2} \ll n_{0} / n_{1}$, we get $\xi^{-2}=$ $k_{B} T L_{x} L_{z}^{2} n_{0}$ and $\zeta=0$, which leads to $\sigma \propto \exp [-2 \xi]=$ $\exp \left[-\left(T_{0}^{\text {low }} / T\right)^{1 / 2}\right]$ with $T_{0}^{\text {low }}=4 /\left(k_{B} L_{x} L_{z}^{2} n_{0}\right)$. Note that, although in this limit the $T$ dependence $(\gamma=1 / 2)$ is determined by the dominating hops in the chain direction, occasional hops between chains will still happen. In the high doping regime, hops in all directions are equally likely and become long ranged at low $T$.

ac conductivity. - In Fig. 2, $\sigma(\omega)$ at room temperature is plotted for three samples with doping levels $0.03<$ $c<0.22$. At low frequencies, the conductivity is seen to be independent of frequency and equal to the dc value. At the onset frequency $\omega_{0} / 2 \pi \approx 1 \mathrm{MHz}$, the conductivity starts to rise, following an approximate power law $\sigma \sim \omega^{s}$ with $s<1$. An extra upturn in the conductivity is observed around a second frequency $\omega_{1} / 2 \pi \approx 10 \mathrm{GHz}$. The temperature dependence of the high frequency $(200-600 \mathrm{GHz})$ conductivity was also measured between 4 and $300 \mathrm{~K}$ (not shown), revealing

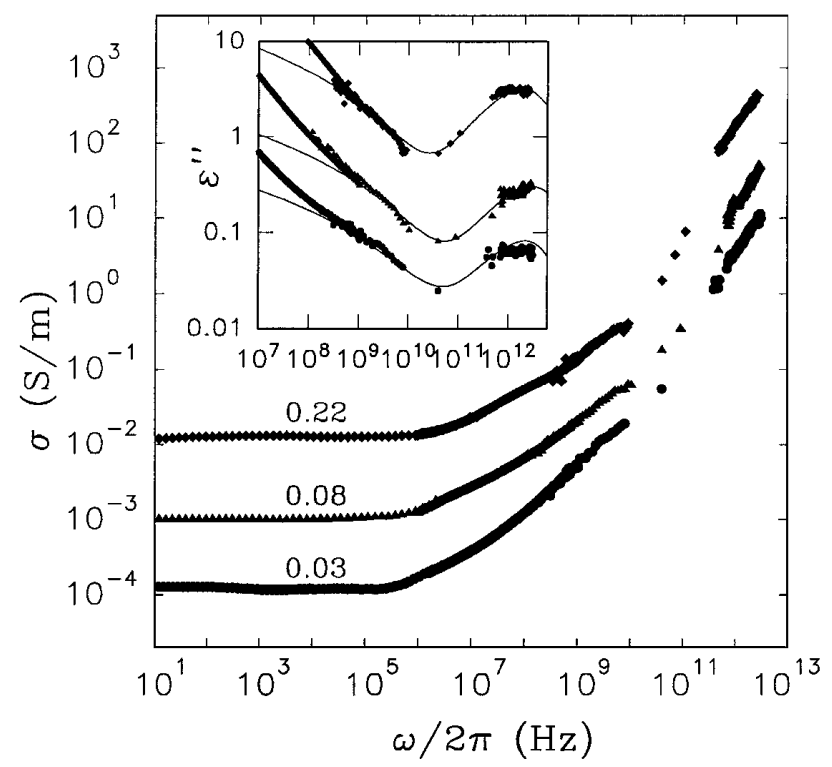

FIG. 2. $\sigma^{\prime}(\omega)$ between $5 \mathrm{~Hz}$ and $3 \mathrm{THz}$ at $300 \mathrm{~K}$ for three doping levels $0.03<c<0.22$. In the inset the loss function $\epsilon^{\prime \prime}(\omega)=\sigma^{\prime}(\omega) / \epsilon_{0} \omega$ is plotted for the same three samples. Drawn lines are fits to Eqs. (2) and (3). Data are corrected for the relatively small dipolar response of the alkoxy side chain (the maximal value of $\epsilon^{\prime \prime}=0.1$ is centered around $0.5 \mathrm{GHz}$ ), most clearly visible in the undoped polymer. that the frequency dependence $\sigma \propto \omega^{s}$ with $s \approx 1.6$ is independent of temperature. The absolute value of the conductivity shows only a weak $(30 \%)$ decrease going from 300 to $150 \mathrm{~K}$, and is constant with temperature below $150 \mathrm{~K}$.

As was discussed above, the conductivity of a system of coupled polymer chains generally consists of contributions due to both inter- and intrachain transport. When the chains are only weakly coupled, i.e., the interchain hopping rate $\Gamma_{\text {inter }}$ is low, the conductivity $\sigma(\omega)$ at frequencies $\omega \gg \Gamma_{\text {inter }}$ is dominated by charge transport processes within single polymer chains. It is widely known that, in a 1D chain, any impurity causes the states to be localized, so the chain has zero conductivity in the limit $\omega \rightarrow 0, T \rightarrow 0$ [21]. At $T \rightarrow 0$ and $\omega>0$, the conductivity of the chain is finite, stemming from resonant photon induced transitions between the localized states, and is at low frequencies $\omega \tau \ll 1$ given by [22]

$$
\sigma_{0}(\omega)=\frac{4}{\pi \hbar b^{2}} e^{2} v_{F} \tau(\omega \tau)^{2} \ln ^{2}(1 / \omega \tau),
$$

where $v_{F}$ is the Fermi velocity on the chain, $\tau$ is the backward scattering time, and $b$ is the interchain separation. Following Ref. [23], this may be written as $\sigma_{0}(\omega)=$ $\left(\pi / 2 b^{2}\right) e^{2} g_{0}^{2} L_{x}^{3} \hbar \omega^{2} \ln ^{2}(1 / \omega \tau)$, where $g_{0}=n_{0} L_{z}^{2}$ is the on-chain DOS per unit length. At finite temperatures, an extra contribution is present due to phonon assisted hopping within the chain. The phonon assisted conductivity is given by the 1D pair approximation [3],

$$
\sigma_{1}(\omega, T)=\frac{\pi^{3}}{128 b^{2}} e^{2} g_{0}^{2} L_{x}^{3} k_{B} T \omega \ln ^{2}\left(\nu_{\mathrm{ph}} / \omega\right),
$$

valid for frequencies $\omega$ below the phonon "attempt" frequency $\nu_{\mathrm{ph}}$. The total conductivity at temperature $T$ is now given by the sum of the two contributions, $\sigma(\omega, T)=\sigma_{0}(\omega)+\sigma_{1}(\omega, T)$ given by Eqs. (2) and (3). The data at $\omega / 2 \pi>10 \mathrm{MHz}$ have been fitted with this $\sigma(\omega)$, as is shown in the inset of Fig. 2. Here, the dielectric loss function $\epsilon^{\prime \prime}(\omega)=\sigma(\omega) / \epsilon_{0} \omega$ is plotted at frequencies between $10 \mathrm{MHz}$ and $3 \mathrm{THz}$, together with the fitting line. The fits are excellent at high frequencies $\omega / 2 \pi>200 \mathrm{MHz}$. The deviations below $200 \mathrm{MHz}$ indicate that either multiple intrachain hopping or interchain transitions have significant contributions in the $\mathrm{MHz}$ regime, which is consistent with the variablerange hopping description at low frequencies.

Parameter values. - From the fits of the ac data, the parameters $\tau=10^{-14} \mathrm{~s}$ and $\nu_{\mathrm{ph}}=2 \times 10^{12} \mathrm{~s}^{-1}$ are extracted. While the phonon frequency $\nu_{\mathrm{ph}}$ is in good agreement with commonly suggested values of $10^{12} \mathrm{~s}^{-1}$ [24], the scattering time $\tau$ is an order of magnitude longer than reported values for other (highly conducting) conjugated polymers $[25,26]$, which is likely due to a smaller $v_{F}$ resulting from the low band filling. The typical time scales now follow directly from the fits, since phonon mediated hops between two sites within a 
chain occur at rates up to $\Gamma_{\mathrm{ph}}=\nu_{\mathrm{ph}} \exp \left[-2 X / L_{x}\right]$. With $X / L_{x} \geq 1$, we have $\Gamma_{\mathrm{ph} \text {, max }} \sim 10^{11} \mathrm{~s}^{-1}$, equivalent to a local diffusion constant $D=10^{-7} \mathrm{~m}^{2} / \mathrm{s}$.

Assuming the localization lengths $L_{x}$ along the chain and $L_{z}$ perpendicular to the chain to be independent of doping level, they can be determined from the combined dc and high frequency conductivity data. From the samples in the low-doping regime, $L_{x}$ and $g_{0}$ can be extracted using $T_{0}^{\text {low }}=4 /\left(k_{B} L_{x} g_{0}\right) \approx 10^{5} \mathrm{~K}$ and Eq. (3). This gives $L_{x}=10 \AA$, indicating that carriers are localized on the chains in regions consisting of two to three rings; furthermore $g_{0}=0.1$ levels $/(\mathrm{eV}$ ring $)$ for $c=0.03$. For the typical hopping distance along the chain, we find $X=\left(L_{x} / 4\right)\left(T_{0}^{\text {low }} / T\right)^{1 / 2}=50 \AA$. At the onset of the high-doping regime $\zeta^{2} \approx 2 n_{0} / n_{1}$; using $Z \approx b$ and $T_{0}^{\text {high }}=64 /\left(k_{B} L_{x} L_{z}^{2} n_{1}\right) \approx 10^{8} \mathrm{~K}$, we find $L_{z}=1.4 \AA$, close to reported values for other conjugated polymers lying deeply in the insulating regime [5,27], and $n_{1}=2 \times 10^{26} \mathrm{eV}^{-1} \mathrm{~m}^{-3}$, implying a DOS of 0.6 states per eV per dopant molecule. Within the localized regions, the carriers move in the chain direction with velocity $v_{x}$, which can be estimated using the fact that in 1D conductors the mean free path $l_{x}=v_{x} \tau \sim L_{x}$; this gives $v_{x} \sim 10^{5} \mathrm{~m} / \mathrm{s}$, similar to values observed in 1D organic conductors [28] and other conjugated polymers [29].

In summary, we measured $\sigma(\omega)$ in a conjugated polythiophene with small interchain overlap. We developed a model that allows a consistent analysis of the $\sigma(\omega)$ data in terms of inter- and intrachain transport. From the low frequency results we have found that carriers are strongly localized on $1 \mathrm{D}$ chains with $L_{z}=1.0 \AA$, and no $3 \mathrm{D}$ metallic islands are present. The high frequency data show 1D transport along the polymer chains with a scattering time $\tau=10^{-14} \mathrm{~s}$, while intrachain phonon assisted hopping proceeds at rates $\Gamma_{\mathrm{ph}} \leq 10^{11} \mathrm{~s}^{-1}$.

It is a pleasure to acknowledge B.F.M. de Waal who prepared the undoped polythiophene samples, G. A. van Albada who assisted in the far-infrared experiments, A. Goossens who performed the Mössbauer measurements, M.P. de Jong and L. J. van IJzendoorn for the induced x-ray analysis, and L. J. de Jongh and O. Hilt who were involved in the discussions. This research is sponsored by the Stichting Fundamenteel Onderzoek der Materie, which is a part of the Dutch Science Organization.

[1] R. Pelster, G. Nimtz, and B. Wessling, Phys. Rev. B 49, 12718 (1994).

[2] L. Zuppiroli et al., Phys. Rev. B 50, 5196 (1994).

[3] Z. H. Wang et al., Phys. Rev. B 42, 5411 (1990); 43, 4373 (1991).

[4] Z. H. Wang et al., Phys. Rev. Lett. 66, 1745 (1991); Phys. Rev. B 45, 4190 (1992).
[5] Q. Li, L. Cruz, and P. Phillips, Phys. Rev. B 47, 1840 (1993).

[6] N. F. Mott, Philos. Mag. 18, 835 (1969).

[7] B. M. W. Langeveld-Voss et al., J. Am. Chem. Soc. 118, 4908 (1996).

[8] A. Goossens (private communication).

[9] Analysis of identically prepared $\mathrm{FeCl}_{3}$ doped poly(paraphenylene vinylene) films with particle induced x-ray emission using a scanning proton microprobe shows overall homogeneous doping distributions with a few disconnected hot spots (typical size a few $\mu \mathrm{m}$ with twice as high Fe concentration) [M. de Jong and L. J. van IJzendoorn, Centre for Plasma Physics and Radiation Technology, Eindhoven University of Technology (private communication)].

[10] Films prepared by drop casting had comparable $\sigma_{\mathrm{dc}}$ values. Powders were more convenient for the experiments performed here.

[11] H. C. F. Martens, J. A. Reedijk, and H. B. Brom, Rev. Sci. Instrum. (to be published); L. J. Adriaanse et al., Phys. Rev. Lett. 78, 1755 (1997).

[12] A. G. Zabrodskii and K. N. Zinov'eva, Sov. Phys. JETP 59, 345 (1984).

[13] B. I. Shklovskii and A. L. Efros, Electronic Properties of Doped Semiconductors (Springer-Verlag, Berlin, 1985).

[14] C. O. Yoon et al., Synth. Met. 75, 229 (1995).

[15] B. Abeles, P. Sheng, M.D. Coutts, and Y. Arie, Adv. Phys. 24, 407 (1975).

[16] D. M. Finlayson and P. J. Mason, J. Phys. C 19, L299 (1986); D. M. Finlayson, P. J. Mason, and I. F. Mohammad, J. Phys. C 20, L607 (1987).

[17] T. G. Castner, in Hopping Transport in Solids, edited by M. Pollak and B. Shklovskii (North-Holland, Amsterdam, 1991) p. 1.

[18] E. P. Nakhmedov, V. N. Prigodin, and A. N. Samukhin, Sov. Phys. Solid State 31, 368 (1989).

[19] A. N. Samukhin, V. N. Prigodin, and L. Jastrabik, Phys. Rev. Lett. 78, 326 (1997); A. N. Samukhin, V. N. Prigodin, L. Jastrabik, and A.J. Epstein, Phys. Rev. B 58, 11354 (1998).

[20] The number of states on a chain is given by $n_{0} \times 2 X L_{z}^{2}$. Therefore the single chain contribution to the density in $(2 X, 2 Z, 2 Z)$ drops by $\left(L_{z} / 2 Z\right)^{2}$.

[21] N. F. Mott and W. D. Twose, Adv. Phys. 10, 107 (1961).

[22] V.L. Berezinskii, Sov. Phys. JETP 38, 620 (1974).

[23] B. I. Shklovskii and A. L. Efros, Sov. Phys. JETP 54, 218 (1981).

[24] H. Böttger and V.V. Bryksin, Hopping Conduction in Solids (Akademie-Verlag, Berlin, 1985).

[25] R. S. Kohlman et al., Phys. Rev. Lett. 78, 3915 (1997).

[26] K. Lee, R. Menon, C. O. Yoon, and A.J. Heeger, Phys. Rev. B 52, 4779 (1995).

[27] M. C. J. M. Vissenberg and M. Matters, Phys. Rev. B 57, 12964 (1998).

[28] G. Soda et al., J. Phys. (Paris) 38, 931 (1977).

[29] B. Beau, J. P. Travers, and E. Banka, Synth. Met. 101, 772 (1999). 